\title{
Transbronchial fine needle aspiration
}

\author{
J LEMER, E MALBERGER, R KÖNIG-NATIV
}

From the Departments of Cardio-thoracic Surgery and Cytology, Rambam Medical Center, Haifa, Israel

ABSTRACT In a pilot study, 21 patients underwent transbronchial fine needle aspiration (TBFNA) using a $45 \mathrm{~cm}-22$ gauge needle guided by means of a semi-rigid metal sleeve, which was introduced $\times$ through a standard rigid bronchoscope. A total of 33 aspirations were performed from main carina (15), paratracheal (five), and lobar carinal (13) foci. Six aspirations yielded malignant cellular is samples, 22 aspirations presented only normal cells, and in five no adequate cellular sample was $\triangle$ obtained. Fifteen patients underwent surgical exploration (mediastinoscopy with or without $ᄋ$ thoracotomy). Four of the cytologically malignant cases were explored and in three the aspiration $\rightarrow$ site was confirmed histologically. In the remaining patients where the site of aspiration was $\rightarrow$ explored, no tumour was demonstrated in the cytologically negative or cytologically inadequate cases. There were no complications from TBFNA. We suggest that TBFNA is useful in determining $\vec{\bullet}$ mediastinal malignant involvement rapidly and with lesser invasion than with current techniques. $N$

The accepted methods of obtaining biopsies from mediastinal and para-mediastinal tissues are based on mediastinoscopy, ${ }^{12}$ anterior mediastinotomy, ${ }^{3}$ thoracotomy and median sternotomy. All these methods involve surgical intervention with its associated risks and expense. In many cases the aim is basically to establish an accurate pathological diagnosis. A method which can provide a pathological diagnosis with lesser invasion is advantageous.

Lately microscope diagnosis of pulmonary and mediastinal malignancies has been obtained by means of transthoracic fine needle aspiration. ${ }^{4}$ However, this method has been used only in those cases in which the lesion was radiologically demonstrated, and it does not provide the surgeon with the information on mediastinal and hilar lymph node pathology which he requires for the assessment of operability.

Based on our diagnostic experience in pulmonary cytology and technical experience in bronchoscopy and transthoracic FNA, we have combined both in a procedure directed towards obtaining cellular material by means of fine needle aspiration through the bronchial or tracheal wall during diagnostic bronchoscopy.

Wang et $a l^{5}$ reported a series of five patients in whom paratracheal masses were aspirated transtracheally using a rigid bronchoscope and an oeso-

Address for reprint requests: Dr J Lemer, Department of Cardio-thoracic Surgery, Rambam Medical Center, Haifa, Israel. phageal varices needle. In his series the lesions were radiologically visible and all five patients had previously undergone non-diagnostic bronchoscopy $\underset{\propto}{\mathbb{Q}}$ or mediastinoscopy or both. In three patients he $\underset{\overrightarrow{2}}{\overrightarrow{2}}$ obtained adequate specimens for cytological $\frac{0}{3}$ examination which revealed tumour cells and large numbers of lymphocytes suggesting the specimens? were from lymph nodes. No further diagnostic procedures were performed on the patients with positive specimens. In one patient with an inadequate $\underset{x}{\stackrel{0}{\mathcal{N}}}$ aspiration the diagnosis was confirmed by trans- $\dot{0}$ thoracic fine needle aspiration of an intraparen -3 . chymal lesion. They suggested that this technique might be considered when subcarinal or submucosal tumour was suspected.

\section{Patients and methods}

A $45 \mathrm{~cm} 22$ gauge needle with a standard Luer hub was used. In view of the needle's flexibility, a special $\widetilde{O}$ hollowed metal guide was designed. The guide is స్ట $43 \mathrm{~cm}$ in length and was made from a length of fine, 0 semi-rigid copper tubing. In order to fix its distate end and to prevent mucosal lacerations, a bulbar

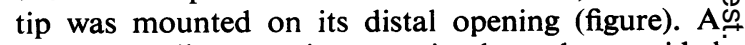
short handle on its proximal end provided manoeuvreability.

The guide, with the needle within its lumen just short of its bulbar tip, is introduced through aब standard rigid bronchoscope during bronchoscopyo under general anaesthesia. The bulbar tip is pushed against the bronchial wall at the site of intendedô 


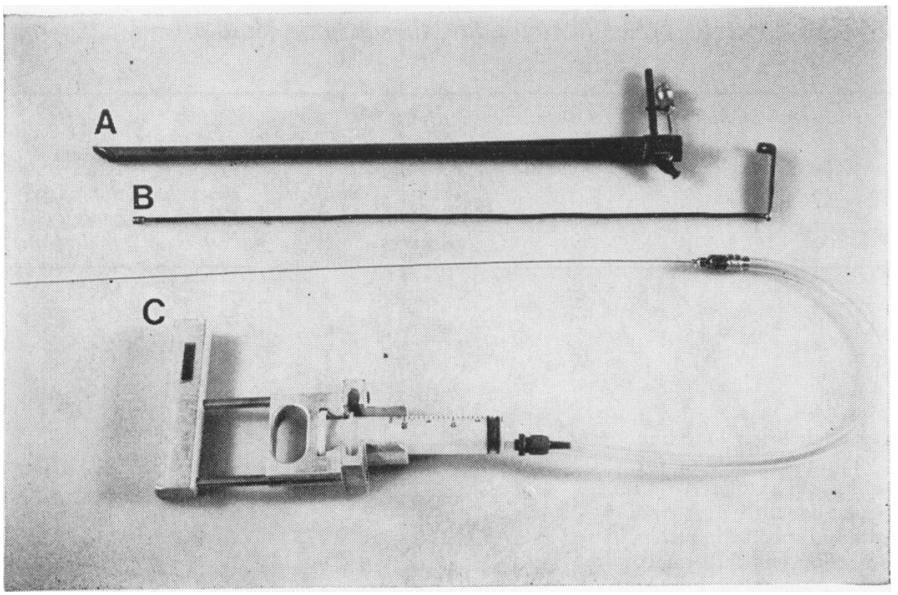

Figure Apparatus for TBFNA. (A)

Standard rigid bronchoscope. $(B)$

Semi-rigid metal sleeve. (C) A $45 \mathrm{~cm}$, 22 gauge needle, PVC connecting tube, and one-hand-grip handle with mounted syringe.

aspiration. Upon fixation of the bulb to the bronchial wall, the needle is advanced $1.0 \mathrm{~cm}$ and the bronchial wall is perforated. A $20 \mathrm{ml}$ disposable plastic syringe, mounted on a one-hand-grip aspiration handle is then attached by means of a $40 \mathrm{~cm}$ flexible PVC connecting tube to the hub of the needle (figure). An assistant exerts negative pressure by retracting the syringe piston, and the bronchoscopist moves the needle to and fro, while its tip is outside the bronchial wall. Before he retracts the needle tip into the bronchial lumen the negative pressure is released. The needle is removed and its contents are expelled on to a glass slide, smeared, and fixed in $96 \%$ ethanol. The smear is later stained by Papanicolaou's method and examined microscopically. If the smear appears inadequate macroscopically, aspiration is repeated at the same site. Before removing the bronchoscope, the sites of aspiration are examined to confirm haemostasis.

Twenty-one patients underwent transbronchial fine needle aspiration (TBFNA). The nature of the procedure was described to the patient and informed consent was obtained.

All patients had radiological findings which were suspicious of malignancy. Of these 21 patients, 14 eventually had histologically or cytologically (exfoliative) proven pulmonary or mediastinal malignancy. In seven cases there was no such evidence. After follow-up periods ranging from 6-12 months, no evidence of malignancy could be demonstrated and it was concluded that the radiological findings were due to non-malignant lesions.

The sites aspirated were beneath the main carina (MC), the paratracheal region (PT), and beneath the lobar carinae (LC). Our policy was to aspirate beneath the main carina in all cases except where the overlying mucosa was abnormal (this would be biopsied).
In the initial stages of our study when the safety and the feasibility of the procedure was being evaluated, we tried to limit the number of aspirations per patient and therefore omitted the aspiration of the main carinal site in some of the cases. The paratracheal site was aspirated when routine radiographs, tomographs, computerised axial tomographs, or bronchoscopy suggested an abnormality. The appropriate lobar carinal site was aspirated in those cases where no cytological or pathological diagnosis was available up to the time of bronchoscopy and when the overlying carinal mucosa was normal. If the carinal mucosa was abnormal or tumour was visible, it was biopsied. The exception to this was the aspiration of a right upper lobe carina which might contribute to the assessment of operability.

A total of 33 aspirations were performed in 21 patients (table 1). In 10 patients one site was aspirated (MC - 5, P-T - 1, LC - 4). In 10 patients two sites were aspirated $(\mathrm{MC}+\mathrm{LC}-7$, $\mathrm{MC}+\mathrm{P}-\mathrm{T}-2, \mathbf{P}-\mathrm{T}+\mathrm{LC}-1)$. In one patient all three sites were aspirated.

Of the 21 patients, 15 underwent mediastinoscopy and of these, five subsequently underwent thoracotomy. In these 15 patients we were able to examine the majority of aspiration sites, and confirm the presence or absence of tumour at that site.

\section{Results (tables 1 and 2)}

In six of the 21 patients who underwent TBFNA, one of the sites of aspiration was cytologically found to contain malignant cells. Of the six, four were explored. All four underwent mediastinoscopythree mediastinoscopy alone and one thoracotomy in addition. The two unexplored cases were considered inoperable clinically and radiologically and any surgical intervention was considered unjustified. 
Table 1 Results of transbronchial fine needle aspiration cytology, mediastinoscopy, thoracotomy, and other investigations in a study of 21 patients

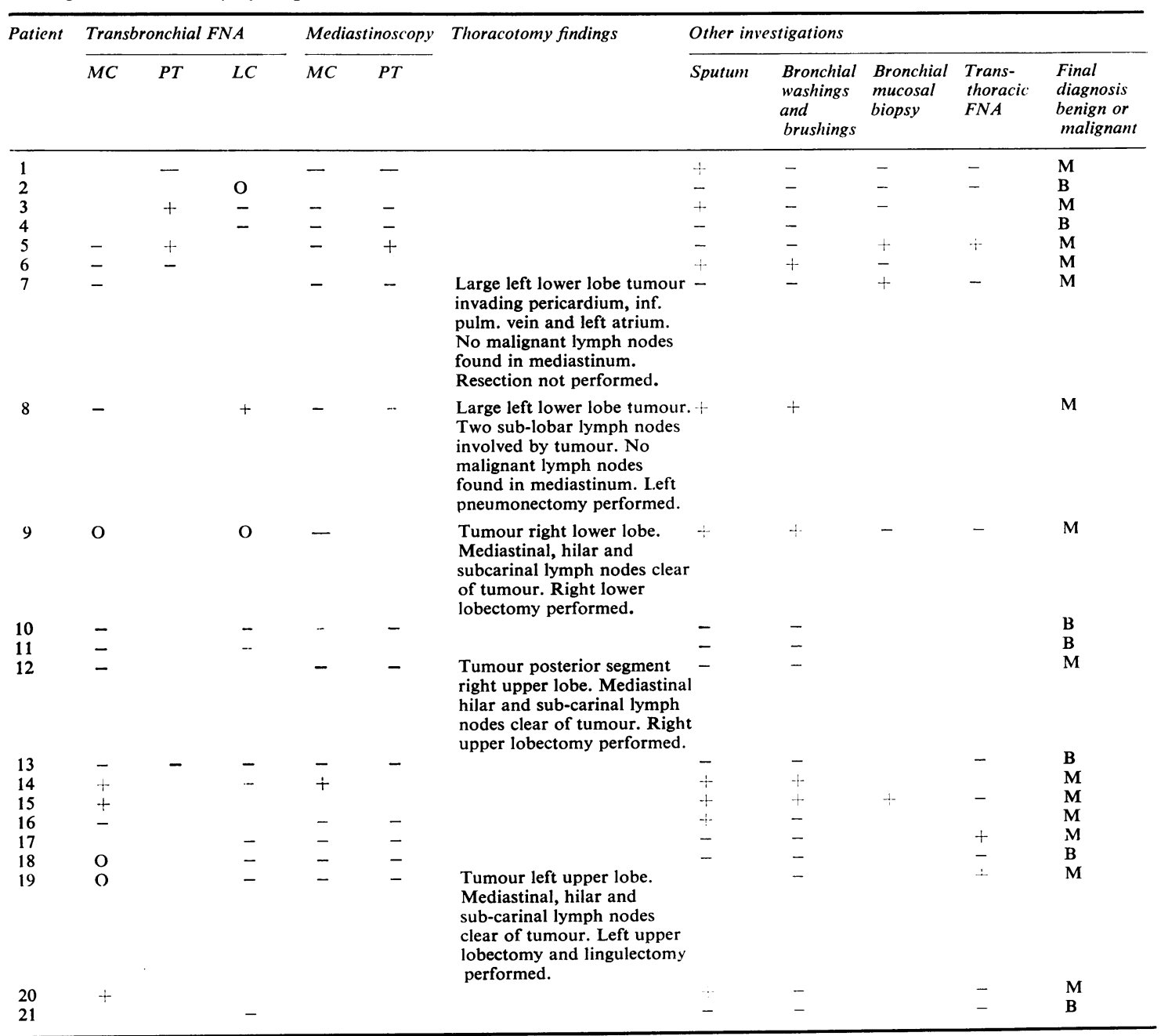

MC = main carina, PT = paratracheal, LC = lobar carina. + Positive for malignancy, - Negative for malignancy, O Cytologically inadequate.

Table 2 Summary of transbronchial fine needle aspiration results in 21 patients

\begin{tabular}{|c|c|c|c|c|c|}
\hline \multicolumn{2}{|l|}{ Positive TBFNA } & \multicolumn{4}{|c|}{ Negative TBFNA } \\
\hline \multirow[t]{2}{*}{ Histologically confirmed } & \multirow[t]{2}{*}{$\begin{array}{l}\text { Histologically } \\
\text { not confirmed }\end{array}$} & \multicolumn{2}{|c|}{$\begin{array}{l}\text { Histologically confirmed tumour free } \\
\text { aspiration site }\end{array}$} & \multicolumn{2}{|c|}{ Aspiration site histologically not confirmed } \\
\hline & & $\begin{array}{l}\text { Cytologically } \\
\text { negative }\end{array}$ & $\begin{array}{l}\text { Cytologically } \\
\text { inadequate }\end{array}$ & $\begin{array}{l}\text { Cytologically } \\
\text { negative }\end{array}$ & $\begin{array}{l}\text { Cytologically } \\
\text { inadequate }\end{array}$ \\
\hline 6 & 3 & 11 & 3 & 3 & 1 \\
\hline
\end{tabular}


Of the four explored cases, in three histopathology confirmed the cytological findings at the site of TBFNA, while in the fourth one mediastinoscopy did not yield malignant tissue.

In the three patients who underwent mediastinoscopy alone, no additional sites of tumour were found apart from the site of positive TBFNA. In the patient who underwent thoracotomy (no 8), a large tumour was present in the left lower lobe. Two sublobar carinal nodes were involved with tumour, but no malignant lymph nodes were found in the mediastinum. The patient underwent left pneumonectomy.

Of the remaining 15 patients, in four the cytological smear in five out of a total of seven aspirations was inadequate (blood or fatty tissue). In the remaining 11 patients who had undergone a total of 16 aspirations, cytology was negative. Eleven of this group of 15 patients underwent mediastinoscopy and of these four subsequently underwent thoracotomy as well. The four unexplored patients relate to three TBFNA negative cases and to one cytologically inadequate case.

In all 11 cases where the site of TBFNA could be explored, no tumour was found when the TBFNA was cytologically negative or inadequate (although seven were known to suffer from pulmonary malignancy). In seven of these 11 patients who underwent mediastinoscopy alone, no additional sites of tumour were found. In the four patients who underwent thoracotomy, no malignant nodes were found at operation in the mediastinum, hilum or at the sublobar carinal site. Three of these patients underwent lobectomy (nos 9, 12, 19). In the fourth patient (no 7) a large tumour of the left lower lobe invaded the pericardium, inferior pulmonary vein and extended into the left atrium. Resection was not attempted. However in this patient no malignant lymph nodes were found in the mediastinum.

\section{Discussion}

The operability of pulmonary malignancies is often determined by the presence or absence of mediastinal lymph node involvement or direct mediastinal invasion by tumour. At present only surgical intervention such as mediastinoscopy or anterior mediastinotomy can determine histological mediastinal involvement before thoracotomy. Both these surgical procedures are not without risk and limitations. Distal main bronchi and lobar carinae as well as some aspects of the trachea are inaccessible to the mediastinoscope and there are also "blind areas" in the mediastinum which cannot be reached by anterior mediastinotomy. Only thoracotomy can establish involvement of sublobar carinal sites.
In our preliminary study TBFNA has no complications whatsoever. There was no chest pain, pneumothorax, or pneumomediastinum after the procedure. Infrequently there was some slight blood-streaking of the sputum. With the experience gained in this study, we feel satisfied that multiple aspirations at suspicious sites in addition to routine aspiration of the submain carinal site are without risk and should be performed.

The bronchoscopic approach provided accessibility to all aspects of the trachea, main stem bronchi, main carina, lobar carinae, and proximal lobar bronchi. In addition, because of the clear landmarks of the bronchial anatomy, accurate localisation of the aspiration site was possible. The relative flexibility of the copper guide enabled us to bend it in order to adapt it to individual cases, and to direct it to otherwise inaccessible sites.

The quality of the cellular aspirates of TBFNA was satisfactory, comparable to FNA samples obtained by standard techniques.

We found good correlation between the cytological findings and the histopathology at the site of aspiration. In our limited study we had no false negative cytology at the site of aspiration. However, the advantage of mediastinoscopy and anterior mediastinotomy over TBFNA is that they allow direct vision whereas TBFNA is essentially a blind procedure. It is therefore conceivable that during mediastinoscopy or anterior mediastinotomy a lymph node suspicious of malignant involvement will be biopsied, whereas that node would not have been aspirated by TBFNA during bronchoscopy. However, in our pilot study this possibility did not occur. We had one TBFNA positive for malignancy whereas mediastinoscopy in that patient was negative. In view of our experience in pulmonary cytology (ex-foliative as well as aspirative) we accepted the cytological finding in that patient. The presence of obvious malignant cells could not be ignored. We tend to believe that in this case mediastinoscopy failed to explore the exact site of TBFNA.

In five of the 33 aspirations no adequate cellular material was obtained. However, it should be pointed out that all the explored cases with an inadequate cytological smear (three cases) proved to be free of tumour at the aspiration site and the fourth case which was not explored was not proven to suffer from malignancy. All malignant sites yielded representative cellular samples. Malignant tissue provides cellular material easily, while aspiration of normal parenchymatous structures yields little, so that we believe that inadequate material on aspiration suggests the absence of malignancy.

A final cytological diagnosis can be made within 20 minutes, or less. Proper handling of a biopsy 
specimen obtained through bronchoscopy or mediastinoscopy usually requires days as the size of the biopsies seldom permits reliable frozen section procedures. Transbronchial fine needle aspiration will yield diagnostic information while the patient is still in the operating theatre.

In one of our non-confirmed cases of positive TBFNA, a case of severe superior vena caval syndrome (no 20), where the trachea was compressed to a slit-like opening, TBFNA gave an immediate cytological diagnosis of malignancy allowing urgent treatment in a patient in whom mediastinoscopy was contraindicated.

We believe that TBFNA will prove of value in establishing the presence of mediastinal malignant involvement, and in assisting the surgeon in the assessment of operability.
We would like to thank the Head of the Department of Cardio-Thoracic Surgery, the Nursing Staff, and the Cytotechnologists for their cooperation.

\section{References}

${ }^{1}$ Carlens E. Mediastinoscopy. Dis Chest 1959;36:343-52.

${ }^{2}$ Pearson FG. An evaluation of mediastinoscopy in the management of presumably operable bronchial carcinoma. J Thorac Cardiovasc Surg 1968;55:617-25.

${ }^{3}$ McNeill TM, Chamberlain IM. Diagnostic anteria mediastinotomy. Ann Thorac Surg 1966;2:532-9.

${ }^{4}$ Dahlgren SE, Nordenstrom B. Transthoracic needle biopsy. Stockholm: Almqvist and Wiksell, 1966.

${ }^{5}$ Wang KP, Terry P, Marsh B. Bronchoscopic needle aspiration biopsy of paratracheal tumors. Am Rev Respir Dis 1978;118:17-21. 